\title{
ULTRASTRUCTURAL RESEARCH OF THE ENDOMETRIUM RECEPTIVITY IN THE CONDITIONS OF PRE-CONCEPTIONAL PREPARATION IN REFRACTORY PREGNANCY LOSS
}

\author{
I. O. Marinkin, D. L. Nepomnyashikh, V. M. Kuleshov, \\ N. A. llizarova, T. O. Semchenko, S. V. Aidagulova \\ NOVOSIBIRSK STATE MEDICAL UNIVERSITY, NOVOSIBIRSK, RUSSIA
}

\begin{abstract}
Background. Refractory pregnancy loss (RPL) is a multifactoral pathology in women of reproductive age which treatment represents great difficulties. Great role in RPL pathogenesis is stipulated by chronic endometritis resulting from disturbances of implantation followed by gestational sac malfunction, which is not less significant than progesterone deficiency.

Objective. The aim of research was to study the dynamics of ultrastructural changes of endometric epithelial cells in the conditions of pre-conception preparation in refractory pregnancy loss.

Methods. 63 samples of endometrium bioptates obtained from female patients of reproductive age with insufficient middle luteal phase of the cycle were studied. Light and electronic microscopy was used to study the morphological changes in endometric epithelial cells.

Results. Pre-conceptional cyclic hormone therapy (1 mg $17 \beta$-estradiol and $20 \mathrm{mg}$ of didrogesteron) in comparison with monotherapy of $20 \mathrm{mg}$ of didrogesteron during 3 months contributed to intracellular regeneration and restoration of secretor phenotype of endometrium epithelial cells corresponding to the status of receptivity with "opened window of implantation".

Conclusions. Cyclic hormonotherapy in combination with complex metabolic therapy as a pre-conceptional preparation contributing to pregnancy illustrates much higher effectiveness of pathogenetically proved preconceptional hormonotherapy
\end{abstract}

KEY WORDS: refractory pregnancy loss, endometrium receptivity, hormone therapy, ultrastructure.

\section{Introduction}

Refractory pregnancy loss (RPL) is a multifactoral pathology in women of reproductive age, which treatment represents great difficulties [1, 3, 4]. In the majority of cases the termination of pregnancy in $P R L$ is due to inadequate reaction to endogenic progesterone and disturbance of secretory transformation of endometrium that results in the disturbance of blastocyst receptivity.

Great role in RPL pathogenesis is stipulated by chronic endometritis resulting from disturbances of implantation followed by gestational sac malfunction, which is not less significant than progesterone deficiency [11]. Weak receptive sensitivity of the endometrium in maintaining normal level of hormones is connected with a wide prevalence of chronic infectious and inflammatory processes accompanied by high content of cytotoxic cells CD16+ and CD56+ and their products such as anti-inflammatory cytokines that, in their turn, contribute to activation of prothrombinase with following thrombosis and placental infarctions [5, 8]. Besides of all mentioned

Address for correspondence: Igor Marinkin, State Budget Educational Institution of Higher Professional Education "Novosibirsk State Medical University" of the Ministry of Public Health, Krasny Prospect, 52, Novosibirsk, 630091, Russia causes of RPL and chromosomal fetus pathologies, there are idiopathic misbirths (approximately 15$25 \%$ ), pathogenesis of which is not clear and empirically progesterone is used [4].

Hormone therapy, as well as drugs possesing direct or indirect action on the steroid metabolism, are used to recover endometrium receptivity in case of correction of luteal insufficiency in RPL [12]. However, a great percentage of misfortune in the recovery of endometrium receptivity and achievement of fertility in RPL needs development of new approaches to the therapy and improvement of schemes of pre-conceptional preparation [9].

Aim of the research: the research was aimed to study the dynamics of ultrastructural changes of epithelial cells of the endometrium in the conditions of pre-conceptional hormone therapy in RPL.

\section{Methods}

Morphological studying of endometrium sampling bioptates in 63 women of reproductive age with $\mathrm{RPL}$ was performed. The patients were empirically divided into 2 groups ( 31 and 32 female patients $27.2 \pm 1.2$ and $28.3 \pm 0.9$ years old, respectively) depending on the scheme of pre-conceptional 
hormone therapy performed during 3 menstrual cycles.

The first scheme is a cyclic hormone therapy, in which femostin was administered 1/10 (1 mg $17 \beta$-estradiola and $10 \mathrm{mg}$ didrogesterone) from the 1 st to the $28^{\text {th }}$ day of the cycle with additional supplementation of dodrogesterone (dufastone) in the dosage $10 \mathrm{mg}$ per 24-hours from the $16^{\text {th }}$ till $25^{\text {th }}$ day. It's important to note that in comparison with recommended schemes [4], the 17 $\beta$-estradiol dosage was decreased twice to stimulate insignificantly proliferative and differentiated activity of cellular endometrium populations. The first scheme included administration of $20 \mathrm{mg}$ of didrogesterone (dufastone) per 24-hours from the $16^{\text {th }}$ to $25^{\text {th }}$ days of cycle. In both groups hormone therapy was used on the background of cyclic metabolic therapy including $100 \mathrm{mg}$ of vitamin $\mathrm{E}$ in combination with $0.012 \mathrm{~g}$ of lipoic acid and $0.1 \mathrm{~g}$ of calcium pantothenate from the $5^{\text {th }}$ to $15^{\text {th }}$ days of the cycle, and with $1 \mathrm{mg}$ of folic acid and $0.5 \mathrm{mg}$ potassium orotate from the $16^{\text {th }}$ to $25^{\text {th }}$ days, respectively.

Sampling bioptates of endometrium were obtained according to the indications on the $22-24^{\text {th }}$ day of the menstrual cycle till pre-conceptional preparation. Bioptates were fixed in $4 \%$ solution of paraform. Paraffin sections colored with haematoxylin and eosin, as well as semithin sections colored with Schiff's reagent and toluydine blue, were studied using microscope Axio Scope.A1 with camera AxioCam MRc5 and software ZEN blue (C.Zeiss). Ultrathin sections of epithelial cells of endometrium contrasted with uranyl acetate and lead citrate were studied with electronic microscope JEM100S. Written agreement on performing examination and treatment of RPL was taken in female patients.

\section{Results}

Before pre-conceptional hormone therapy the complex of structural changes of endometrium in female patients with RPL in the middle secretory stage of menstrual cycle in light-optical studying of paraffin and semithin sections corresponded to general pathological agreement about severe dystrophy and atrophy. Endometrial glands were reduced in the amount, being shortened and polymorphic according to the maturity: secretory glands did not prevail and were desynchronized with the phase of menstrual cycle, with dystrophy and atrophy of epithelium. Subepithelial stroma of endometrium was characterized by weak signs of decidualization of cellular elements and the presence of simple subepithelial lymphatic cells (fig. 1), that didn't correspond to the severe inflammatory changes in chronic endometritis.

Electronic microscopic examination of epithelial cells of endometrium revealed ultrastructural signs of a considerable reduction of the level of biosynthetic processes. The decreasing of height of epithelial cells, apical membrane of which didn't form pinopods, associated with severely decreased amount of nucleolus and components of canalicular system in nucleus and was accompanied with the reduction of elements of granular cytoplasmatic net in the absence of mega mitochondria. The decrease of synthesized protein and energetic potentials of epithelial cells was due to the accumulation of only singular glycogen grains.

Thus, ultrastructural equivalents of the disturbance of endometrium receptivity in RPL were insufficiently expressed by elements of protein synthesized complex of the nucleus and cytoplasm that in combination with little content of glycogen and absence of mega mitochondria confirmed insufficiency of plastic and energetic material for providing of secretory transformation. Disbalance of hormonedependent cellular differentiation of epithelial cells of endometrium in cyclic stage in female patients with RPL is possible to be interpreted as systemic manifestation of universal syndrome of regenerative and plastic insufficiency [7], which essence consists of the disturbance of processes of intracellular regeneration and forming of plastic deficiency in different organs and tissues undergoing chronic unfavorable influences.

The use of the $1^{\text {st }}$ scheme of hormone therapy during 3 menstrual cycles has stimulated intracellular regenerative reactions of epithelial cells since an adequate stage of the cycle by means of differentiation and expression of secretor phenotype of endometrium (fig. 2) more than proper level of reproductive health with "opened window of implantation" in comparison with the $2^{\text {nd }}$ group (fig. 3 ). Gigantic mega mitochondria contributing to hormone-induced cytological differentiation were revealed in the epithelial cells of endometrium. There were large karyosomes with clasters of regular tubular structures, those membranes were appropriate to the tubules of granular cytoplasmatic net with numerous polysomes in the nucleus of the majority of cells (fig. 4); gross deposits of glycogen were localized in the cytoplasm diffusively and like super nuclear aggregates. High polymorphic protrusions of apical plasmolemma as pinopods were formed in $47-63 \%$ of epithelial cells (fig. 5), other cells had polymorphic microvillus and cilia on their apexes.

\section{Discussion}

As a result of using the 2 nd scheme of preconceptional preparation during three cycles, it was noted the growth of proliferative activity of epithelial cells which corresponds to the luteal phase of the menstrual cycle - the glands of stellar and serrated forms with wide lumens, and folded counters lined 
by prismatic epithelium with ultrastructural signs of adequate cytodifferentiation. However, taking into account the complex of clinical data we can observe that the given scheme in most cases did not provide the stimulation expression level of plastic and energy potential of endometrial epithelial cells necessary to "open a window to implantation".

Normally the epithelium and the endometrium stroma are rapidly renewing tissues under the influence of a particular combination of reproductive hormones and should be exposed to remodeling for the normal receptivity of blastocytes. The basis of luteal phase deficiency is a reduced level of progesterone receptor expression as a consequence of inadequate estrogen content [6]. However, the morphological "immaturity" of endometrium can be observed even during the insufficient progesterone production as a result. What is important is not only the level of steroids in the organism, but also the adequate realization of hormonal effect $[8,10]$.

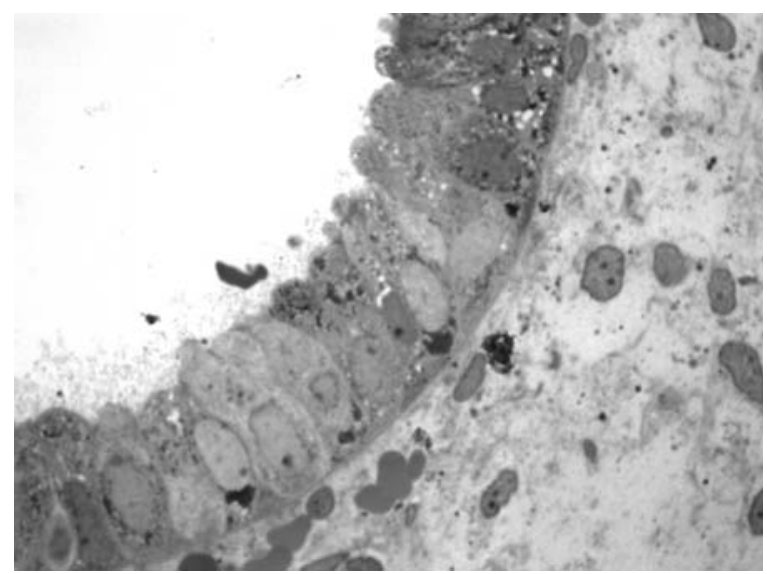

Fig 1. Refactory pregnancy loss. Endometrial biopsy sample on the 23rd day of menstrual cycle. Dystrophy of epithelial cells, absence of pinopods; isolated stromal cells. Semifine section, coloring with Schiffs reagent and toluidine blue. Magnification 1050

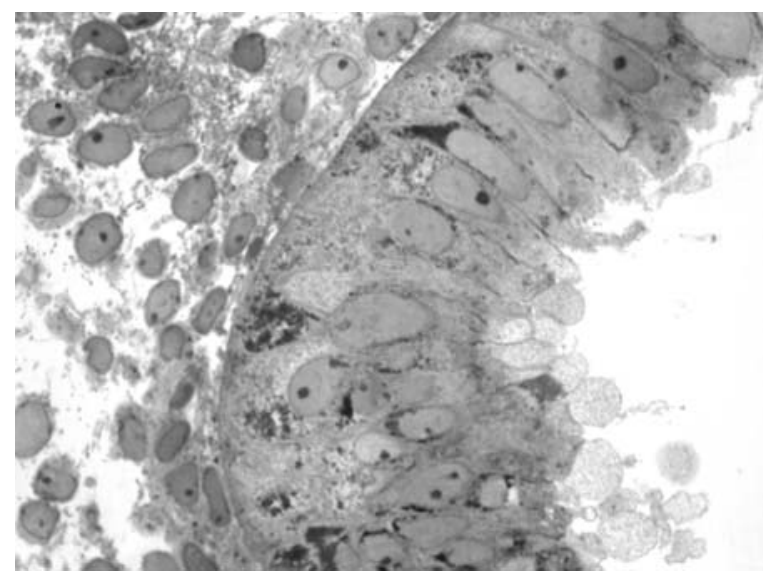

Fig. 2. Refactory pregnancy loss after cyclic hormonotherapy during 3 months. Endometrium biopsy sample on the 23d day of menstrual cycle. Numerous pinopods on the apical pole of epithelial cells. Semifine section, coloring with Schiffs reagent and toluidine blue. Magnification 1050.

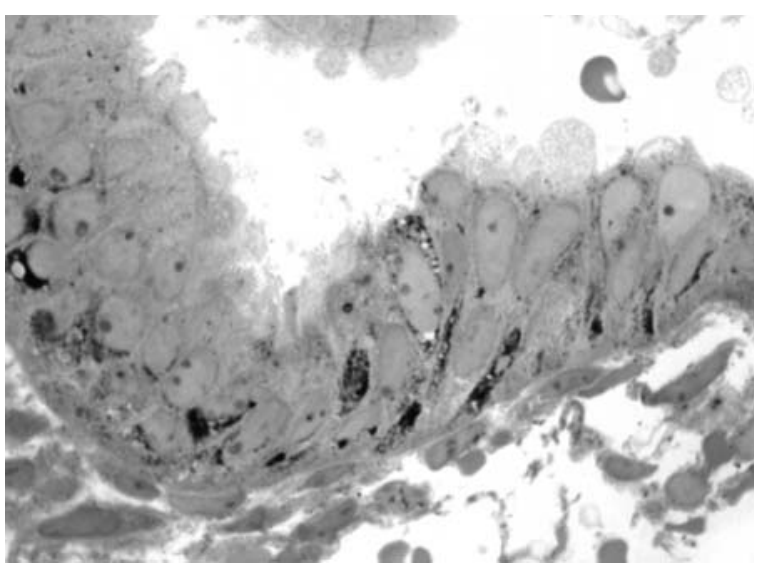

Fig. 3. Refactory pregnancy loss after dydrogesterone hormonotherapy during 3 months. Endometrium biopsy sample on the $24^{\text {th }}$ day of menstrual cycle. Isolated pinopods on the epical poles of epithelial cells. Semifine section, coloring with Schiffs reagent and toluidine blue. Magnification 1050.

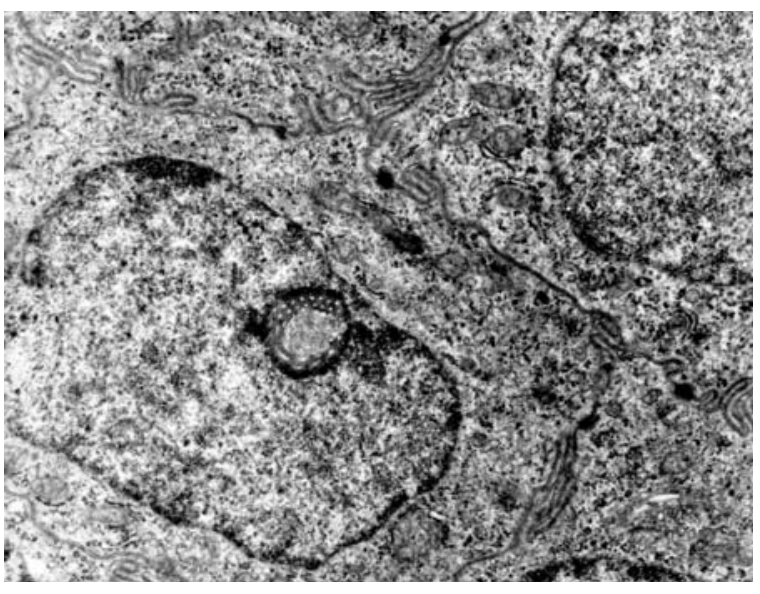

Fig. 4. Refactory pregnancy loss after cyclic hormonotherapy during 3 months. Fragments of endometrium of granulocytes on the 23rd day of menstrual cycle: the nucleolus in the nucleus is with the clusters of granular tubular structures; the cytoplasm contains free and attached polysoms. Electrondiffraction pattern. Magnifying power 10000 .

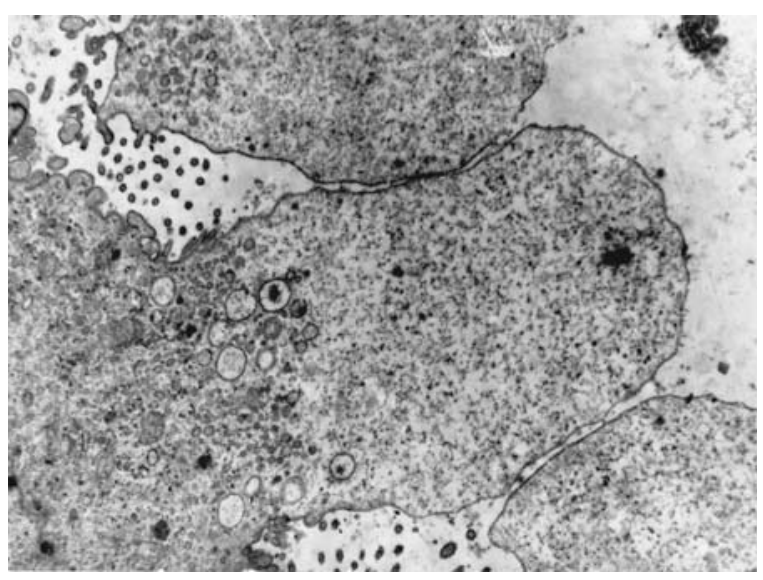

Fig. 5. Refactory pregnancy loss after cyclic hormonotherapy during 3 months. High pinopods on the apical poles of endometrium epithelial cells on the 23rd day of menstrual cycle: numerous exosomes and vesicles. Electron-diffraction pattern. Magnifying power 15000 . 
In our trial the low doses of estrogens at the stage of pre-conceptional preparation of patients with refectory pregnancy loss contributed both to proliferation and hormone-mediated epithelium differentiation with receptors expression both to estrogens and to progesterone with the increased dosages to the $16^{\text {th }}$ day of a cycle inducing secretory transformation. In other words, the cyclic hormonotherapy, providing the adequate supply of reproductive steroid hormones, consequently induced the intracellular regeneration of endometrium epithelial cells with the restoration of the necessary level of the receptor apparatus to estrogens and progesterone. The result of this process was the realization of status of endometrium receptivity corresponding to the indexes of reproductive health.

Mucous membranes including endometrium play a leading role in the adaptation processes; complex morphological studies of mucous membrane based on endoscopic biopsies demonstrate the permanence in their reaction that allows to formulate the conception of "reaction unity of mucous membranes" frequently realized by the syndrome of regenerative and plastic insufficiency based on the reducing of the processes of intracellular regeneration [2]. This syndrome can become a fundamental basis to create principally new approaches to the therapy of chronic diseases especially of endometrium by means of remedies inducing regenerative reactions in opposition to aggressive antibiotic therapy [3].

\section{References}

1. Milovanov AP, Gerasimova OP. Clinical and morphological control of the efficiency of pregravid treatment in women after non-developing pregnancies. Obstetrics and Gynecology 2013; 5: 87-90.

2. Непомнящих Г. И. Биопсия бронхов: Морфогенез общепатологических процессов в легких. М. : Изд-во РАMН; 2005: 384.

3. Radzinskiy VE, Dimitrova V.I, Mayskova IU. Nondeveloping pregnancy. Manual. M.; 2009: 200.

4. Sidelnikova VM. Habitual pregnancy loss. M.; 2005: 303.

5. Sukhikh GT, Vanko LV. Immunology of pregnancy. M.; 2003: 400 .

6. Khmelnitskiy OK. Pathomorphological diagnostics of gynecological diseases. S-Petersburg; 1994: 480.

7. Tsellarius UG, Semenova LA, Nepomnyashchikh LM et al. Favorites fundamental problems of myocardial histopathology Bul. of Sib. Div. of RAMN 1982; 3: 22-29.

\section{Conclusions}

Thus, the obtained results allow us to distinguish a new aspect in morphogenesis of endometrium pathology in case of refractory pregnancy loss. In the complex of interrelated pathologic phenomena, there are the endocrine disorders, thrombophilia, and chronic endometritis. One of the important positions is concerned with endometrium dystrophy, which is evident in the reduction of intracellular regenerative epithelium reactions. The ultrastructural equivalents of reducing the endometrium receptivity is the reduction of protein synthesizing nuclear and cytoplasmic organelles in epithelial cells that underlie the discrepancy of the structure of luteal phase cycle with a reduction of secretory activity and "closed door to implantation".

The strategy basis of refractory pregnancy loss treatment is the intensification of regenerative reactions in cellular endometrium populations that contributed to the induction of biosynthetic reactions with endometrium receptivity and fertility. Cyclic hormonotherapy in combination with the complex metabolic therapy as a pre-conceptional preparation contributed to pregnancy in $16(51.6 \%)$ women of the $1^{\text {st }}$ group and $13(40.6 \%)$ women in the $2^{\text {nd }}$ group; full-term children with Apgar scores 8-9 were born in the $1^{\text {st }}$ group - in 10 women $(32.3 \%)$, in the $2^{\text {nd }}$ group - in 8 women $(25.0 \%)$ demonstrating higher effectiveness of pathogenetically proved preconceptional hormonotherapy.
8. Badawy AM, EI-Gharib ME, Zalata K. Should endometrial biopsy be a routine investigation for recurrent early-pregnancy loss? Gynaecol. Endosc 2002; 11 (6): 397-399.

9. Ford HB, Schust DJ. Recurrent pregnancy loss: etiology, diagnosis, and therapy. Rev. Obstet. Gynecol. 2009; 2 (2): 76-83.

10. Lea R.G., Oliver S.S. Immunoendocrine aspects of endometrial function and implantation. Reproduction. 2007; 134 (3): 389-404.

11. Prado-Drayer A, Teppa J, Sanchez P, Camejo MI. Immunophenotype of peripheral T-lymphocytes, NK cells and expression of CD69 activation marker in patients with recurrent spontaneous abortions during the mid-luteal phase Amer. J. Reprod. Immunol 2008; 60 (1): 66-74.

12. Szekeres-Bartho J, Balasch J Progestagen therapy for recurrent miscarriage. Hum. Reprod. Update 2008; 14 (1): 27-35. 